\title{
Signe de Kehr. Un signe clinique qui peut sauver !
}

\section{Kehr's Sign. A Clinical Sign that can Save!}

\section{H. Slimani $\cdot$ S. Hugonnot $\cdot$ L. Sengler}

Reçu le 8 décembre 2016; accepté le 15 mars 2017

(C) SFMU et Lavoisier SAS 2017

Un patient de 59 ans, hypertendu, sans autre antécédent notable, était admis aux urgences suite à un malaise décrit comme lipothymique, associé à une douleur scapulaire gauche caractérisée par son accentuation à l'inspiration profonde. L'interrogatoire précisait en outre l'absence de traumatisme associé. À l'admission, le patient était conscient, orienté et apyrétique ; la pression artérielle était à $85 / 45 \mathrm{mmHg}$, la fréquence cardiaque à 108 battements par minute et une saturation en oxygène à $96 \%$ en air ambiant. L'examen clinique retrouvait une pâleur cutanéomuqueuse, l'abdomen était souple avec une sensibilité diffuse à la palpation profonde, mais sans défense retrouvée. Le toucher rectal était indolore et sans trace de sang visible. L'examen de l'épaule gauche était sans particularité. Le bilan sanguin initial a retrouvé un syndrome inflammatoire (la protéine C-réactive à $61 \mathrm{mg} / \mathrm{l}$ et une hyperleucocytose à $19500 / \mathrm{mm}^{3}$ ), un taux de lactates à $4,8 \mathrm{mmol} / \mathrm{l}$, une hémoglobine à $7,2 \mathrm{~g} / \mathrm{dl}$, un taux de prothrombine à $78 \%$ et une créatininémie à $166 \mu \mathrm{mol} / 1$ (soit un débit de filtration glomérulaire estimé à $39 \mathrm{ml} / \mathrm{min}$ ), sans troubles hydro-électrolytiques ni perturbation du bilan hépatique. Un TDM thoraco-abdominal réalisé en urgence objectivait un volumineux hémopéritoine associé à un hématome périsplénique faisant orienter vers une rupture de la rate (Fig. 1). Une splénectomie d'hémostase en urgence a été réalisée dont les suites ont été simples.

La rupture atraumatique de rate est une entité clinique rare dont la mortalité reste élevée. Dans une revue systématique de la littérature entre 1980 et 2011 concernant 845 patients, le taux s'élevait à 12,2\% [1]. La présentation clinique est peu spécifique, rendant son diagnostic parfois difficile. La présence d'une douleur de l'hypocondre gauche avec irradiation scapulaire gauche, appelée signe de Kehr, a une valeur d'orientation intéressante [2]. Ce signe fut décrit par le chirur-

\footnotetext{
H. Slimani $(\bowtie) \cdot S$. Hugonnot $\cdot$ L. Sengler

Service des urgences, hôpital Nord-Franche-Comté,

100 route de Moval, CS 10499 Trevenans,

F-90015 Belfort, France

e-mail : hakim.slimani@hnfc.fr
}

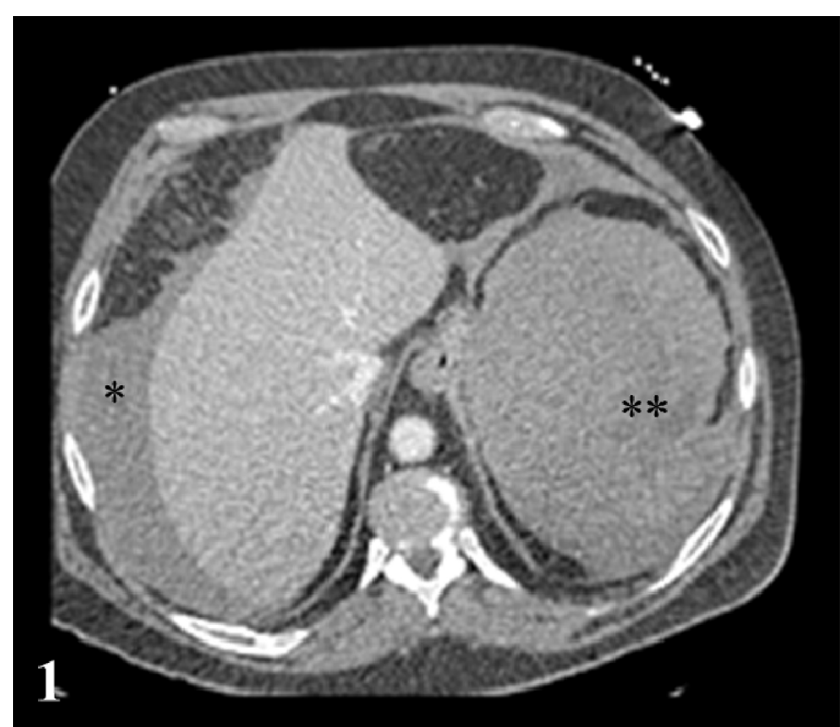

Fig. 1 Tomodensitométrie abdominale, coupe transversale. * : épanchement intra-abdominal périhépatique. $* *$ : saignement actif sur le versant externe de la capsule splénique

gien allemand Hans Kehr (1862-1916). Il s'agit d'un exemple classique de douleur projetée. Une irritation du diaphragme (par une atteinte d'un organe de proximité) est transmise par le nerf phrénique avec une douleur venant se localiser dans la région supraclaviculaire homolatérale. Cette projection est expliquée par l'origine radiculaire cervicale commune ( $\mathrm{C} 3$ et C4) du nerf supraclaviculaire et du nerf phrénique. Ainsi, l'association d'une douleur scapulaire et d'une instabilité hémodynamique sans orientation étiologique évidente doit inciter le praticien à rechercher un épanchement intrapéritonéal.

\section{Références}

1. Gedik E, Sadullah G, Aldemir M, et al (2008) Non-traumatic splenic rupture: report of seven cases and review of the literature. World J Gastroenterol 14:6711-6

2. Klimpel V (2004) Does Kehr's sign derive from Hans Kehr? A critical commentary on its documentation? Chirurg 75:80-3 